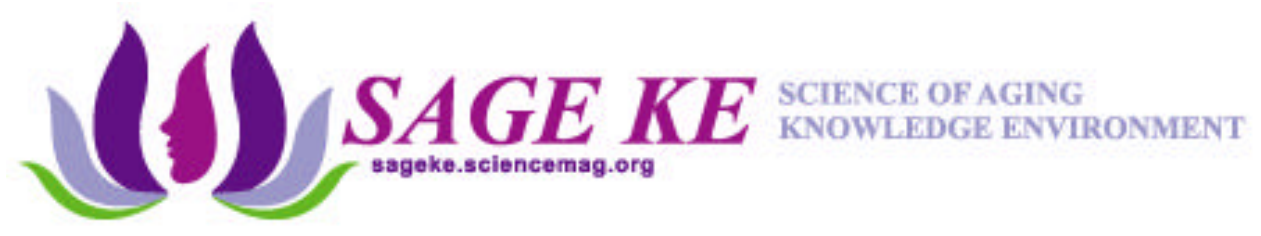

October 3, 2001

\title{
Using Yeast to Discover the Fountain of Youth
}

\author{
Matt Kaeberlein, Mitch McVey and Leonard Guarente \\ The authors are in the Department of Biology, Massachusetts Institute of Technology, Cambridge, MA \\ 02139, USA. E-mail: keeshond@mit.edu (M.K.)
}

Document URL: http://sageke. sciencemag.org/cgi/content/full/sageke;2001/1/pe1

\section{Introduction}

The budding yeast Saccharomyces cerevisiae has long served as a model organism for the study of basic cellular processes such as transcription, cell cycle regulation, and DNA repair. Its short generation time, well-established molecular genetics, and fully sequenced genome have made this organism a favorite of researchers in diverse fields. Much of the information obtained has been shown to apply to higher eukaryotes, including humans. Recently, researchers have begun using yeast to tackle one of the outstanding questions in science: How and why do organisms age? The identification of individual genes in yeast that can affect the aging process itself has elevated this single-celled fungus to full contender status in the aging field.

The foundation of the study of yeast aging was laid when Mortimer and Johnston discovered that yeast cells have a finite life-span that can be measured (1). Since then, more than 30 genes have been implicated in yeast aging (see SAGE KE's Genes/Interventions Database), and at least one molecular mechanism of aging has been established. In this Perspective, we present two fundamentally different measures of aging in yeast: replicative life-span and stationary phase survival (chronological life-span). We describe the benefits and limitations of each and present models that attempt to explain these "aging" phenomena. Finally, we present compelling evidence that the use of yeast as a model system will ultimately prove beneficial to the study of human aging.

\section{Replicative Life-Span}

The budding yeast $S$. cerevisiae reproduces by asymmetric cell division, giving rise to an "old" mother cell and a "young" daughter cell. The replicative life-span of a yeast cell is defined as the number of daughter cells produced by a given mother before senescence. In practice, replicative life-span is measured by removing daughter cells from their mothers by micromanipulation after each division and the tallying the number of daughters produced by each mother (life-span assay). The viability curve for a population of yeast cells measured in this way closely resembles viability curves for other organisms, including humans, and this observation suggests that replicative life-span represents a true aging process (Fig. 1). 


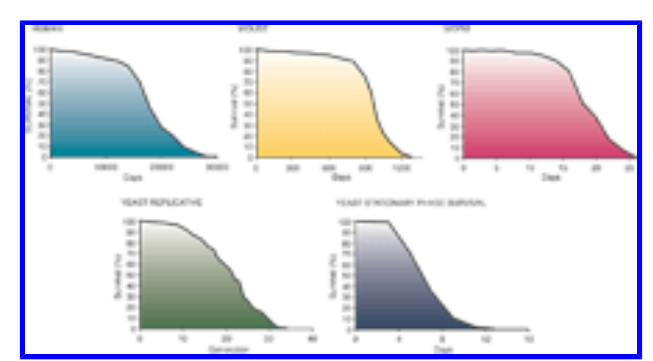

View larger version $(18 \mathrm{~K})$ :

[in this window]

[in a new window]
Fig. 1. Viability curves from different model organisms have a similar, characteristic shape. Representative mortality data are shown for Homo sapiens (23), Mus musculus (23), and Caenorhabditis elegans (16). Two different types of aging, replicative (ㅁ) and postdiauxic survival (11, and see section on postdiauxic survival), are shown for S. cerevisiae.

The usefulness of yeast as a model system for replicative aging was highlighted by a recent study that demonstrated one of the first molecular causes of aging in any organism. Sinclair and Guarente (2) found that senescence can be caused by the formation and accumulation of extrachromosomal ribosomal DNA (rDNA) circles (ERCs) in mother cells. ERCs are formed by homologous recombination between tandemly arrayed chromosomal rDNA repeats. These circular molecules are self-replicating and asymmetrically segregate to the mother cell, resulting in an exponential increase in ERC copy number with age.

There are several lines of experimental evidence that support a causal role for ERCs in the aging process of yeast. First, mutations that increase the rate of ERC accumulation, such as mutations in the NADdependent histone deacetylase SIR2, result in a shortened life-span (3). Sir2p promotes silencing at the $H M$ loci, telomeres, and rDNA and inhibits rDNA recombination. Second, mutations that decrease the rate of ERC accumulation, such as the deletion of FOB1, extend life-span (4). Fob1p is required for the formation of a unidirectional replication fork block in the rDNA. In the absence of Fob1p, rDNA recombination and ERC formation are reduced approximately 10-fold. Taken together, these results demonstrate that ERCs are both necessary and sufficient for wild-type aging. Although it is unclear whether ERCs contribute to aging in other eukaryotes, SIR2 appears to control longevity in higher organisms (see below).

The ERC model has recently been challenged in two reports. First, Heo et al. (5) showed that the shortlived $s g s 1$ mutant fails to accumulate ERCs more rapidly than wild-type mother cells. This was interpreted to suggest that ERCs do not cause aging. However, the authors failed to distinguish between a mutation that shortens life-span by accelerating the normal aging process and a mutation that shortens life-span by causing a defect unrelated to the normal aging process. In fact, as discussed below, mutation of SGS1 has recently been shown to result in a high rate of stochastic age-independent mortality ( $\underline{6})$, which suggests that most sgs 1 mother cells are not dying from old age. A second result that has been purported to weaken the ERC model is the claim that mutation of SIR 2 does not cause an increase in ERC levels ()). In this case, the authors examined ERC levels in an unsorted, logarithmically growing culture of yeast cells. However, when ERC levels were examined in an aged population of mother cells, mutation of SIR 2 was clearly shown to cause a significant increase in ERC accumulation (프).

\section{Postdiauxic Survival: A Model for Postreplicative Senescence?}

Yeast aging can also be defined in a chronological sense, based on the ability of cells to maintain viability over time in a nondividing state. When grown on a rich medium such as yeast extract, peptone, and dextrose, yeast cells continue to divide until they have exhausted the nutrient supplies in the medium. At this point, they greatly alter their metabolism and enter a hypometabolic, nondividing state known as stationary phase. The amount of time that cells remain viable in this nondividing state can last on the order of several weeks. When grown on a synthetic medium with glucose as the sole carbon source, yeast 
undergo a similar cessation of replication; however, they continue to maintain a high metabolic rate. Cell survival in this postdiauxic state is dramatically reduced relative to stationary phase survival. Interestingly, a postdiauxic survival curve is similar in shape to a plot of replicative life-span (Fig. 1) and is clearly nonstochastic in nature. This similarity suggests that these two distinct processes may both be pertinent to aging studies.

Postdiauxic survival most closely resembles the aging of postmitotic, differentiated cell populations within a multicellular organism. A large body of research suggests that accumulated oxidative damage caused by reactive oxygen species (ROS) (such as hydrogen peroxide and superoxide anion) is a key determinant of organismal life-span (see The Two Faces of Oxygen). Treatment with antioxidants has been shown to extend the life-span of Caenorhabditis elegans ( $\underline{8})$, and neuronal overexpression of enzymes that detoxify oxygen radicals promotes longevity in flies (9). Likewise, there is evidence to suggest that ROS may limit postdiauxic survival in yeast. One of the primary metabolic changes that occurs upon diauxic shift is a switch from fermentation to respiration. This leads to a concomitant increase in the production of ROS and the associated cellular damage. Detoxification of ROS by superoxide disumutase is required for postdiauxic survival, and overexpression of human Bcl-2 (which stimulates antioxidant protection) increases the survival of wild-type yeast cells $(\underline{10})$.

Recent work suggests that yeast postdiauxic survival may provide a useful model for ROS-induced mortality. By screening for mutants that were resistant to oxidative stress by paraquat, Fabrizio et al. (111) isolated two $S$. cerevisiae strains that showed increased postdiauxic survival. Mutations in two genes, $C Y R 1$ and $S C H 9$, were responsible for the increased viability. $C Y R 1$ codes for adenylate cyclase and is an activator of adenosine 3',5'-monophosphate (cAMP)-dependent protein kinase (PKA) activity. Sch9p is a protein kinase with significant homology to C. elegans AKT-1 and AKT-2, which function downstream of the longevity regulator DAF-2 in the insulin-like signaling pathway.

In addition to postdiauxic survival, there is also some evidence to suggest that ROS may play a role in the replicative aging process. For example, mutation of $C Y R 1$ also extends replicative life-span by a mechanism resembling caloric restriction (12). However, in the case of replicative life-span, the effects of $C Y R 1$ are likely to be independent of the stress response pathway activated by MSN2 and MSN4. Msn2p and Msn4p are zinc-finger transcription factors required for transcriptional activation of many stress response genes in yeast. In contrast, the increased postdiauxic survival caused by mutation of $C Y R I$ requires MSN2 and MSN4 (11), suggesting that $C Y R 1$ may function by two different mechanisms to regulate both types of aging in yeast. A link between replicative aging and oxidative stress also was suggested by Laun et al. (13), who showed that mutation of the superoxide dismutases or catalases of yeast can reduce replicative life-span. Of course, as with any mutation that shortens life-span, it remains to be determined whether this represents a true acceleration of the normal aging process or is simply the result of a detrimental mutation. The critical test of this hypothesis will be to discover an intervention or genetic manipulation that reduces ROS and also extends replicative life-span.

Another interesting parallel between the life-span of nondividing yeast and replicative aging was uncovered by the observation that yeast cells passaged through stationary phase have a reduced replicative life-span (14). Furthermore, the longer the cells are grown in stationary phase, the shorter their life-span after a return to logarithmic growth. Whether this suggests a common mechanism of aging for postmitotic and mitotic cells is not clear. It will be interesting to discover whether mutations that extend replicative capacity also increase the life-span of postdiauxic cells. In any case, it appears that yeast will be useful as a model to further test the link between ROS and aging and to determine whether similar genetic determinants are important for both replicative life-span and postdiauxic survival.

\section{Yeast Provides Insight About Aging in More Complex Organisms}

Critics of the yeast aging field maintain that, as opposed to other model organisms such as C. elegans and 
mice, yeast is unlikely to yield useful information regarding aging in humans (15). Many argue that the primary cause of aging in multicellular organisms is not the loss of replicative potential, and that the study of replicative senescence in yeast is therefore irrelevant. Those of us who work in the field obviously disagree with this assessment and have been vindicated recently by a series of stunning findings. These results suggest a surprising degree of conservation in the aging process, or at least in the genes that control it, among distantly related organisms.

The most impressive correlation between aging in yeast and higher eukaryotes comes from the work of Tissenbaum and Guarente (16), who found that overexpression of a SIR2 homolog in C. elegans extends life-span in that organism (Fig. 2). Two years earlier, it was shown that overexpression of SIR2 extends the replicative life-span of yeast cells (3). Strikingly, the mechanism by which SIR2 extends life-span in each organism appears to be different. The $C$. elegans SIR 2 counterpart, sir-2.1 acts in the insulin-like signaling pathway known to control aging in worms (16). In yeast, $S I R 2$ silences gene expression and inhibits recombination in the rDNA repeats. The picture that emerges is that although distinct mechanisms may be at the heart of aging in different organisms, they could still fall under SIR 2 control. It is possible that the nicotinamide adenine dinucleotide (NAD)-dependent histone deacetylase activity of Sir2 proteins ( $\underline{17})$ allows them to connect metabolic rate to regulation of aging mechanisms in a wide array of contexts. The involvement of SIR2 in extending yeast life-span by caloric restriction is consistent with this hypothesis.

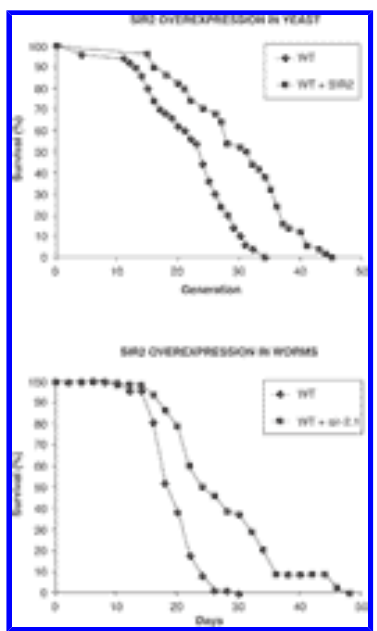

View larger version $(7 \mathrm{~K})$ :

[in this window] [in a new window]
Fig. 2. A longevity-promoting gene from yeast also functions to extend life-span in higher eukaryotes. Overexpression of SIR2 extends life-span in $S$. cerevisiae (3). Overexpression of the SIR2-homolog Sir-2.1 extends life-span in C. elegans (16).

Yeast also has served as a useful model for studying the role of RecQ helicases in aging. S. cerevisiae has a single RecQ family member, SGS1. Mutation of SGS1 results in a shortened life-span and premature onset of some age-associated phenotypes such as sterility, relocalization of Sir3p to the nucleolus, and nucleolar fragmentation (18). Likewise, mutation of a human RecQ homolog, WRN, results in Werner syndrome, a condition characterized by symptoms of premature aging (see Of Hyperaging and Methuselah Genes). The Sgs1 helicase has been shown to be important for the survival of yeast cells that are unable to maintain their telomeres (19). Because most human cells also suffer from the problem of shrinking telomeres, the results in yeast suggest that some of the characteristics of cell lines derived from patients with Werner syndrome may be due to defects in telomere maintenance. This view is bolstered by the finding that the expression of telomerase in these cells can rescue them from premature senescence (20). In addition, it was demonstrated recently that the short life-span of yeast sgs 1 mutants may in large part be caused by cell 
cycle arrest due to the action of DNA damage checkpoint mechanisms ( $\underline{6})$. Further dissection of the cellular role of Sgs $1 p$ in yeast should allow the formulation of experiments that determine the function of the RecQ helicases in humans.

As mentioned above, postdiauxic survival in yeast may also provide a useful model for the aging of postmitotic cells. The link between ROS and aging in higher organisms is well established. A model for ROS-induced mortality in yeast would give researchers in the field of aging a valuable tool for dissecting the genetic and biochemical pathways involved in this process.

Finally, caloric restriction, which has long been known to extend the life-span of mice, has now been shown to do so in yeast $(\underline{12}, \underline{21})$ and is allowing the elucidation of the genetics behind this life-span extension. Early studies of aging in yeast implicated the cAMP/PKA pathway as an important determinant of longevity. Mutation of the guanosine triphosphate-binding Ras 1 protein increases life-span by up to $40 \%$, and overexpression of Ras $2 p$ has a similar effect (22). The mechanism by which the Ras proteins act to regulate longevity is not understood. However, Lin et al. (12) recently demonstrated that caloric restriction (through the growth of yeast on low-glucose medium) extends life-span by up to $50 \%$ and that this effect can be mimicked by mutations that decrease signaling through the cAMP/PKA pathway. Perhaps these early life-span-extending experiments with the Ras proteins were tapping into this PKAmediated caloric restriction pathway. As mentioned above, life-span extension by caloric restriction requires Sir2p, which suggests that this protein may play a general role in the relationship between lifespan and metabolism.

\section{Conclusions}

Researchers who use yeast to study aging must certainly be pleased with the events of the past few years. Not only are we beginning to decipher how various biological components interact to regulate life-span in our favorite fungus, but we are now obtaining the first hints that at least some of these pathways are also important determinants of the rate of aging in distantly related organisms. One task that remains is to determine which of the pathways are specific to yeast aging and which are conserved among more complex organisms. The genetic and biochemical dissection of those that are conserved may someday lead to the development of therapies that allow human beings to lead longer and healthier lives.

October 3, 2001

\section{Comment on Article}

\section{Further Reading}

- For related information generated by a computer algorithm, click on Related I nfo in the left navigation bar.

- To view related information on SAGE KE selected by the editors, click on Editors' Choice in the left navigation bar or scroll to the bottom of the page.

\section{References}

1. R. K. Mortimer, J. R. Johnston, Life-span of individual yeast cells. Nature 183, 1742-1751 (1959).

2. D. A. Sinclair, L. Guarente, Extrachromosomal rDNA circles--a cause of aging in yeast. Cell 91, 10331042 (1997). [Medline] 
3. M. Kaeberlein, M. McVey, L. Guarente, The SIR2/3/4 complex and SIR2 alone promote longevity in Saccharomyces cerevisiae by two different mechanisms. Genes Dev. 13, 2570-2580

(1999).[Abstract/Free Full Text]

4. P. A. Defossez, R. Prusty, M. Kaeberlein, S. J. Lin, P. Ferrigno, P. A. Silver, R. L. Keil, L. Guarente, Elimination of replication block protein Fob1 extends the life-span of yeast mother cells. Mol. Cell 3, 447455 (1999).[Medline]

5. S. J. Heo, K. Tatebayashi, I. Ohsugi, A. Shimamoto, Y. Furuichi, H. Ikeda, Bloom's syndrome gene suppresses premature ageing caused by Sgs1 deficiency in yeast. Genes Cells 4, 619-625 (1999). [Medline] 6. M. McVey, M. Kaeberlein, H. A. Tissenbaum, L. Guarente, The short life-span of Saccharomyces cerevisiae sgsl and srs 2 mutants is a composite of normal aging processes and mitotic arrest due to defective recombination. Genetics 157, 1531-1542 (2001).[Abstract/Free Full Text]

7. S. Kim, A. Benguria, C. Y. Lai, S. M. Jazwinski, Modulation of life-span by histone deacetylase genes in Saccharomyces cerevisiae. Mol. Biol. Cell 10, 3125-3136 (1999).[Abstract/Free Full Text]

8. S. Melov, J. Ravenscroft, S. Malik, M. S. Gill, D. W. Walker, P. E. Clayton, D. C. Wallace, B. Malfroy, S. R. Doctrow, G. J. Lithgow, Extension of life-span with superoxide dismutase/catalase mimetics. Science 289, 1567-1569 (2000).[Abstract/Free Full Text]

9. J. P. Phillips, T. L. Parkes, A. J. Hilliker, Targeted neuronal gene expression and longevity in Drosophila. Exp. Gerontol. 35, 1157-1164 (2000).[CrossRef][Medline]

10. V. D. Longo, L. M. Ellerby, D. E. Bredesen, J. S. Valentine, E. B. Gralla, Human Bcl-2 reverses survival defects in yeast lacking superoxide dismutase and delays death of wild-type yeast. J. Cell. Biol. 137, 1581-1588 (1997).[Abstract/Free Full Text]

11. P. Fabrizio, F. Pozza, S. D. Pletcher, C. M. Gendron, V. D. Longo, Regulation of longevity and stress resistance by Sch9 in yeast. Science 292, 288-290 (2001).[Abstract/Free Full Text]

12. S. J. Lin, P. A. Defossez, L. Guarente, Requirement of NAD and SIR2 for life-span extension by calorie restriction in Saccharomyces cerevisiae. Science 289, 2126-2128 (2000).[Abstract/Free Full Text] 13. K. U. Frohlich, M. Breitenbach, Aged mother cells of Saccharomyces cerevisiae show markers of oxidative stress and apoptosis. Mol. Microbiol. 39, 1166-1173 (2001).[CrossRef][Medline]

14. K. Ashrafi, D. Sinclair, J. I. Gordon, L. Guarente, Passage through stationary phase advances replicative aging in Saccharomyces cerevisiae. Proc. Natl. Acad. Sci. U.S.A. 96, 9100-9105

(1999).[Abstract/Free Full Text]

15. H. Gershon, D. Gershon, The budding yeast, Saccharomyces cerevisiae, as a model for aging research: a critical review. Mech. Ageing Dev. 120, 1-22 (2000).[CrossRef][Medline]

16. H. A. Tissenbaum, L. Guarente, Increased dosage of a sir-2 gene extends lifespan in Caenorhabditis elegans. Nature 410, 227-230 (2001).[CrossRef][Medline]

17. S. Imai, C. M. Armstrong, M. Kaeberlein, L. Guarente, Transcriptional silencing and longevity protein Sir2 is an NAD-dependent histone deacetylase. Nature 403, 795-800 (2000).[CrossRef][Medline]

18. D. A. Sinclair, K. Mills, L. Guarente, Accelerated aging and nucleolar fragmentation in yeast $s g s 1$ mutants. Science 277, 1313-1316 (1997).[Abstract/Free Full Text]

19. F. B. Johnson, R. A. Marciniak, M. McVey, S. A. Stewart, W. C. Hahn, L. Guarente, The Saccharomyces cerevisiae WRN homolog Sgs1p participates in telomere maintenance in cells lacking telomerase. EMBO J. 20, 905-913 (2001).[Abstract/Free Full Text]

20. F. S. Wyllie, C. J. Jones, J. W. Skinner, M. F. Haughton, C. Wallis, D. Wynford-Thomas, R. G. Faragher, D. Kipling, Telomerase prevents the accelerated cell ageing of Werner syndrome fibroblasts. Nature Genet. 24, 16-17 (2000).[CrossRef][Medline]

21. J. C. Jiang, E. Jaruga, M. V. Repnevskaya, S. M. Jazwinski, An intervention resembling caloric restriction prolongs life-span and retards aging in yeast. FASEB J. 14, 2135-2137

(2000).[Abstract/Free Full Text]

22. J. Sun, S. P. Kale, A. M. Childress, C. Pinswasdi, S. M. Jazwinski, Divergent roles of RAS1 and RAS2 in yeast longevity. J. Biol. Chem. 269, 18638-18645 (1994).[Abstract/Free Full Text]

23. D. Sinclair, K. Mills, L. Guarente, Aging in Saccharomyces cerevisiae. Annu. Rev. Microbiol. 52, 533-560 (1998).[CrossRef][Medline]

24. We thank P. Fabrizio and V. Longo for providing data used in Fig. 1 and H. Tissenbaum and T. Kaeberlein for helpful discussion and comments. 


\section{Comment on this article:}

\section{Read all Comments}

comments on Yeast and the Fountain of Youth

David Gershon

SAGE KE, 11 Oct 2001 [Full text]

Re: comments on Yeast and the Fountain of Youth

Matt Kaeberlein

SAGE KE, 25 Oct 2001 [Full text]

The sir 2 overexpression and knockout

Florian Muller

SAGE KE, 28 Oct 2001 [Full text]

Re: The sir 2 overexpression and knockout

Matt Kaeberlein

SAGE KE, 29 Oct 2001 [Full text]

Re: Re: Re: The sir 2 overexpression and knockout

Charles R Fred

SAGE KE, 19 Nov 2001 [Full text] 